\title{
On the well-mixed condition and consistency issues in hybrid Eulerian/Lagrangian stochastic models of dispersion
}

\author{
4 Meïssam L. Bahlali* . Christophe \\ 5 Henry - Bertrand Carissimo
}

7 Received: DD Month YEAR / Accepted: DD Month YEAR

\begin{abstract}
We clarify issues related to the expression of Lagrangian stochastic models used for atmospheric dispersion applications. Two aspects are addressed: the respect of the well-mixed criterion and the correspondence between Eulerian and Lagrangian turbulence models when they are combined in practical simulations. In particular, it is recalled that the fulfillment of the well-mixed criterion depends only on the proper incorporation of the mean pressure-gradient term as the mean drift term of the Langevin equation. New consistency issues between duplicate fields within Eulerian/Lagrangian hybrid formulations are also brought out, especially regarding turbulence models, boundary conditions, and divergence-free condition. Such hybrid methods, where mean flow quantities calculated with an Eulerian approach are provided to the Lagrangian approach, are commonly used in atmospheric dispersion simulations for their numerical efficiency. Nevertheless, it is shown that serious inconsistencies can result from coupling Eulerian and Lagrangian models that do not correspond to the same level of description of the fluid turbulence.
\end{abstract}

* Meissam L. Bahlali, corresponding author.

Department of Civil and Environmental Engineering, Imperial College London, London, UK

CEREA, Joint laboratory Ecole des Ponts ParisTech/EDF R\&D, 6 quai Watier, 78401 Chatou Cedex, France

E-mail: meissambahlali@gmail.com

This research was done while M. L. Bahlali was a Ph.D. student at CEREA, in 2015-2018.

C. Henry

Laboratoire Lagrange, Université de la Côte d'Azur, OCA, CNRS, 06304 Nice Cedex 4, France

INRIA Sophia Antipolis, Méditerranée, 2004 route des Lucioles - BP 93, 06902 Sophia Antipolis Cedex, France

B. Carissimo

CEREA, Joint laboratory Ecole des Ponts ParisTech/EDF R\&D, 6 quai Watier, 78401

Chatou Cedex, France 
Table 1 List of abbreviations

\begin{tabular}{|cc|cc|}
\hline CFD & Computational Fluid Dynamics & SDE & Stochastic Differential Equation \\
PDE & Partial Differential Equation & SLM & Simplified Langevin Model \\
PDF & Probability Density Function & & \\
\hline
\end{tabular}

Keywords Dispersion models · Eulerian/Lagrangian methods · Stochastic modeling · Turbulence · Well-mixed criterion

\section{Introduction}

Lagrangian stochastic models for atmospheric dispersion purposes have been studied extensively since the 1980s. These models reproduce convective transport in the atmosphere by explicitly tracking a large number of representative fluid particles where, usually, the evolution of particle velocities is driven by a stochastic differential equation of a Langevin type. Such equation includes two terms, respectively known as the drift vector and the diffusion matrix, which are to be determined. The diffusion matrix is defined following Kolmogorov's similarity theory and the main closure issue is therefore the expression of the drift.

First well-known references in Lagrangian stochastic modeling are, for example, Wilson et al. (1981); Sawford (1985); Van Dop et al. (1985); De Baas et al. (1986), or the classic paper Thomson (1987). In most of these works, the so-called 'well-mixed condition' issue was brought out as a challenging issue. First introduced by Sawford (1986), this issue corresponds to the thermodynamic constraint stating that an initially uniform distribution of fluid particles (each representing an equal amount of mass) in an incompressible flow must remain uniform. The well-mixed criterion is a necessary criterion for any Lagrangian stochastic model to be regarded as acceptable since its violation means that density is no longer constant (therefore at variance with the incompressibility constraint). For that reason, various authors have tried to provide conditions to ensure that it is respected. It was shown by Sawford and Guest (1988) and Borgas et al. (1997) that certain flow properties need to be accounted for, see Wilson and Sawford (1996). Historically in the atmospheric literature, authors adopted other selection criteria and worked on a case-by-case basis (homogeneous or inhomogeneous turbulence, Gaussian or non-Gaussian turbulence hypothesis, convective, stable or neutral atmosphere, etc.). As a result, a confusion surrounding expressions of the drift term in the Langevin equation has sometimes led to non well-mixed models that made various authors adopt a back-and-forth philosophy and add ad-hoc new terms to make up for the problem (Lin and Gerbig 2013).

Since the works mentioned above, significant improvements in Lagrangian stochastic modeling have been achieved in other fields where similar models are currently applied, in particular in turbulent combustion modeling (Pope 1985,1991 ), in single-phase flow turbulence modeling (Pope 1994b) as well as in dispersed turbulent two-phase flows applications (Minier and Peirano 2001; 
Minier 2015). For instance, Pope (1987) showed that a necessary and sufficient condition for the well-mixed criterion to be fulfilled is that the calculated mean velocity field satisfies the continuity equation and that this condition is, in turn, equivalent to incorporating properly the mean pressure gradient term in the Langevin model. However, in the recent atmospheric literature relative to Lagrangian dispersion modeling (see, e.g., Franzese (2003), Stohl et al. (2005), Alessandrini and Ferrero (2009), Tinarelli et al. (2013)), the formulations introduced by Pope and referred to as 'probability density function (PDF) methods' are not widely used. Cassiani et al. (2005a,b, 2007) referred to Pope's PDF framework for micro-mixing modeling but followed Thomson (1987)'s approach to close the fluctuating pressure gradient and viscous terms.

In this context, the present study aims at bridging the developments made in Lagrangian stochastic models in reactive and two-phase flows with atmospheric dispersion applications. It has a two-fold objective: (a) to clarify remaining confusions about the well-mixed condition; (b) to provide a better understanding of consistency issues between fields obtained from Eulerian and Lagrangian simulations when hybrid Eulerian/Lagrangian numerical formulations are used.

The paper is organized as follows: in Sect. 2, Lagrangian stochastic models for atmospheric dispersion are introduced and the formulation of the wellmixed condition, as well as consistencies between duplicate turbulence models and wall-boundary conditions, are presented. To illustrate how consistent formulations can be developed in the context of hybrid Eulerian/Lagrangian frameworks, the details of such a model are briefly provided. Numerical assessment of the respect of the well-mixed criterion through the simple introduction of the mean pressure gradient is detailed in Sect. 3, through three simulations of non-homogeneous turbulent flows that cover a wide range of practical situations. More specifically, numerical results obtained in three standard cases are used to illustrate two main points:

(i) The satisfaction of the well-mixed criterion depends only on the proper introduction of the mean pressure-gradient term as the mean drift component of the Langevin model, regardless of further details.

(ii) In so-called Eulerian/Lagrangian hybrid numerical formulations, such as that considered in present simulations, the mean fields corresponding to the first and second moments of the velocity field are duplicate fields and need to be regarded as such. In other words, identity between predictions of the Eulerian and Lagrangian solvers must be verified for a model and its numerical implementation to be acceptable.

\section{Particle Stochastic Methods: Theory and Numerics}

This section provides a brief description of the key concepts and issues behind particle stochastic methods. Interested readers are referred to existing comprehensive reviews for more details (Monin and Yaglom 1971; Pope 1985, 1994a, 2000; Minier and Peirano 2001; Minier 2016). 
Table 2 Two existing formulations of the SLM

\begin{tabular}{ll}
\hline Model written in terms of: & Formulation \\
\hline Instantaneous velocity & $d U_{p, i}=-\frac{1}{\rho_{f}} \frac{\partial\left\langle P_{f}\right\rangle}{\partial x_{i}} d t-\frac{U_{p, i}^{\prime}}{T_{L}} d t+\sqrt{C_{0} \epsilon_{f}} d W_{i}$ \\
Fluctuating velocity & $d U_{p, i}^{\prime}=\left(\frac{\partial\left\langle U_{f, i}^{\prime} U_{f, j}^{\prime}\right\rangle}{\partial x_{j}}-U_{p, j}^{\prime} \frac{\partial\left\langle U_{f, i}\right\rangle}{\partial x_{j}}\right) d t-\frac{U_{p, i}^{\prime}}{T_{L}} d t+\sqrt{C_{0} \epsilon_{f}} d W_{i}$ \\
\hline
\end{tabular}

\subsection{The Simplified Langevin Model}

A simplified Langevin model (SLM) is used (more details can be found in Pope (1985, 2000); Minier and Peirano (2001)), with this Lagrangian stochastic model describing the dynamics of (fluid) particles by explicitly tracking their position $X_{p}$ and velocity $U_{p}$ according to,

$$
\begin{aligned}
& d X_{p, i}=U_{p, i} d t \\
& d U_{p, i}=-\frac{1}{\rho_{f}} \frac{\partial\left\langle P_{f}\right\rangle}{\partial x_{i}} d t-\frac{U_{p, i}-\left\langle U_{f, i}\right\rangle}{T_{L}} d t+\sqrt{C_{0} \epsilon_{f}} d W_{i} .
\end{aligned}
$$

The first term on the right-hand side (r.h.s.) of Eq. 1b corresponds to the mean pressure gradient (with $\rho_{f}$ the fluid density and $\left\langle P_{f}\right\rangle$ the mean pressure of the fluid). In the second term on the r.h.s., the particle velocity relaxes toward the local mean fluid velocity taken at the particle location, i.e. $\left\langle U_{f, i}\right\rangle=\left\langle U_{f, i}\right\rangle\left(t, \mathbf{X}_{p}(t)\right)$. The time scale for this relaxation is $T_{L}$, which represents the Lagrangian integral time scale $T_{L}=\frac{k_{f}}{\epsilon_{f}}\left(\frac{1}{2}+\frac{3}{4} C_{0}\right)^{-1}$. In the last term, $d W_{j}$ are independent Wiener processes, $C_{0}$ a constant given by Kolmogorov's theory, and $\epsilon_{f}$ the mean turbulence kinetic energy dissipation rate at the particle position. It should be noted here that the simplified Langevin model is appropriate to high Reynolds number and the effect of viscosity is absent (Pope 2000). The viscous effect being of primary importance in the viscous sublayer, additional terms are usually included in near-wall models (see for example Sect. 2.2.2 or Wacławczyk et al. (2004) for near-wall viscosity effects in Lagrangian PDF methods). Such models can be used for simple boundary flows such as the channel flow (see Sect. 3.2) but are more problematic in complex geometries as the flow behind an obstacle (see Sect. 3.3). The viscous issues are still open and will be investigated in the future.

Another type of formulation for the SLM has been derived using the fluctuating velocity $U_{p, i}^{\prime}$, defined as $U_{p, i}^{\prime}=U_{p, i}-\left\langle U_{f, i}\right\rangle$. The difference between these two types of formulation for the SLM are summarized in Table 2 and interested readers are referred to, e.g., Minier et al. (2014). It can be seen that both formulations are similar (blue terms are identical) and there is no intrinsic difference since a simple change of variables allows to go from one formulation to the other. Yet, there are significant differences in the expression of the mean pressure gradient: whereas it appears directly in the formulation written with instantaneous velocities, it includes the derivative term of the 
Reynolds-stress tensor in the formulation written with fluctuating velocities. We wish to emphasize here that ignoring this term results in spurious drift effects (see Minier et al. (2014) for more details). As a result, the proper account of the mean pressure-gradient term is key in ensuring the well-mixed condition. In the following, only the formulation written with instantaneous velocities is considered.

\subsection{Hybrid Eulerian/Lagrangian Simulations}

In the following, we resort to a hybrid Eulerian/Lagrangian calculations where the fluid phase is simulated using an Eulerian solver and the particle phase is solved with a Lagrangian one. The use of such hybrid formulations requires a specific attention for the terms entering the particle dynamics, such as the mean fluid velocity at the particle location (see Eq. 1). In the following, the issues related to the consistency of the Eulerian and Lagrangian models are detailed.

\subsubsection{Consistency Issues on Turbulence Models}

If the proper introduction of the mean pressure gradient on the r.h.s. of Eq. 1 is enough to ensure that the mean Navier-Stokes equation derived from the PDF model is correctly retrieved, the specific details of a Langevin model (i.e., the last two terms on the r.h.s. of Eq. 1b) come into play when considering the second-order velocity moments or, in other words, the transport equations for the Reynolds-stress components. The connection between Lagrangian stochastic models was first developed in Pope (1994b) and has been addressed in detail in several works, cf. Pope (2000); Minier and Peirano (2001); Minier et al. (2014).

The derivation of the Reynolds-stress equations from a Langevin model, such as the SLM in Eq. 1, reveals that for each formulation of a Langevin model there is a corresponding second-order, or $R_{i j}-\epsilon$, turbulence model. This connection is of importance, first to indicate that Lagrangian stochastic are true second-order turbulence models when they are used as stand-alone approaches and, second, to point out a consistency issue when they are used in hybrid Eulerian/Lagrangian numerical formulations. In particular, an SLM for the Lagrangian solver is consistent with a Rotta second-order turbulence model for the Eulerian solver (with the Rotta constant deriving from the value taken from the $C_{0}$ constant). More details are provided in Chibbaro and Minier (2011) and Minier $(2015,2016)$. It should be noted here that variants of the present hybrid Eulerian/Lagrangian formulation could be developed to match other models for the Eulerian solver (for instance using LES or Reynolds stress equation models). In that sense, the present formulation serves as an illustration of how consistent hybrid Euler/Lagrange formulations can be developed. 


\subsubsection{Consistency Issues on Boundary Conditions}

Another issue is the formulation of wall-boundary conditions in particle stochastic approaches, which is a subject that has received very little attention. For instance, a first idea consists in applying specular rebound: every particle that crosses a wall boundary and exits the computational domain is reflected back with the same streamwise velocity component as the incoming one, i.e. $U_{p, x}^{\text {in }}=U_{p, x}^{\text {out }}$ and $U_{p, y}^{\text {in }}=U_{p, y}^{\text {out }}$. Yet this amounts to writing a zero-flux boundary condition for the streamwise velocity component, which fails to account for the momentum exchange with the wall. Following the same spirit of the one used to derive so-called wall-function boundary conditions in computational fluid dynamics (CFD) codes, where a boundary condition is applied in the logarithmic region near the wall rather than at the wall itself (cf. Pope (2000)), a better representation of wall-boundary conditions to capture the constantstress boundary layer is (assuming that $z$ is the wall-normal direction)

$$
\begin{aligned}
& U_{p, x}^{\text {in }}=U_{p, x}^{\text {out }}-2 \frac{\left\langle U_{f, x}^{\prime} U_{f, z}^{\prime}\right\rangle}{\left\langle U_{f, z}^{\prime 2}\right\rangle} U_{p, z}^{\text {out }}, \\
& U_{p, y}^{\text {in }}=U_{p, x}^{\text {out }}-2 \frac{\left\langle U_{f, y}^{\prime} U_{f, z}^{\prime}\right\rangle}{\left\langle U_{f, z}^{\prime 2}\right\rangle} U_{p, z}^{\text {out }}, \\
& U_{p, z}^{\text {in }}=-U_{p, z}^{\text {out }},
\end{aligned}
$$

where the subscript 'in' stands for the particle's incoming velocity value and 'out' the outgoing one (see Fig. 1). The rationale behind these expressions for particle-based stochastic models are detailed in Dreeben and Pope (1997) and Minier and Pozorski (1999).

Such formulations are needed to reproduce the correct behavior of the mean streamwise fluid velocity component in wall boundary layers. In hybrid Eulerian/Lagrangian approaches, they also represent an additional consistency issue since wall-boundary conditions are actually formulated twice, in the Eulerian and in the Lagrangian solvers. Consistent wall-boundary conditions are important for the correct computation of particle second-order moments near walls as will be seen later in the case of an infinite channel flow (see Sect. 3.2).

\subsubsection{Correction on particle velocities}

In stand-alone approaches, the mean pressure is calculated as the constraint needed to have $\operatorname{div}\left(\left\langle\mathbf{U}_{p}\right\rangle\right)=0$ (cf. Minier and Pozorski (1999)). In hybrid Eulerian/Lagrangian formulations, it is easier to use directly the mean pressure gradient calculated by the Eulerian solver, say $P^{E}$, in the Langevin equations, so that Eq. 1b can be expressed as

$$
d \widetilde{U}_{p, i}=-\frac{1}{\rho_{f}} \frac{\partial P^{E}}{\partial x_{i}} d t+d M_{i}
$$

where $\left\langle d M_{i}\right\rangle=0$, and where the notation $\widetilde{U}_{p, i}$ is used to indicate that this is a first estimation of particle velocities and where $d M_{i}$ stands for the fluctuating 


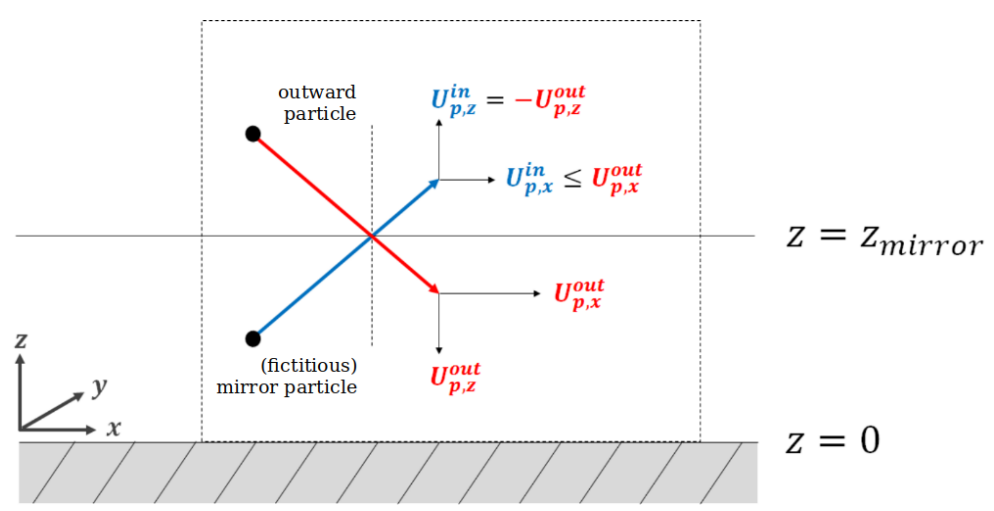

Fig. 1 Sketch of the wall/particle interaction showing how particles arriving at $z=z_{\text {mirror }}$, which is chosen in the logarithmic layer, are reflected back in the domain so as to respect a zero-mass flux in the wall-normal direction as well as the momentum exchange due to friction in the longitudinal direction. $\mathbf{U}_{p}^{i n}$ (oriented upward) and $\mathbf{U}_{p}^{\text {out }}$ (oriented downward) stand for the inward and outward velocities, respectively

part of the Langevin models, which is not relevant to the present discussion. There are, however, always small differences between the numerical predictions of $\left\langle\mathbf{U}_{f}\right\rangle(t, \mathbf{x})$ and $\left\langle\widetilde{\mathbf{U}}_{p}\right\rangle(t, \mathbf{x})$. Consequently, Eq. 3 means that the resulting mean velocity field obtained from the Lagrangian solver $\left\langle\widetilde{\mathbf{U}}_{p}\right\rangle(t, \mathbf{x})$ is only approximately a divergence-free field. A more precise implementation consists in writing

$$
d U_{p, i}=-\frac{1}{\rho_{f}} \frac{\partial P^{E}}{\partial x_{i}} d t-\frac{1}{\rho_{f}} \frac{\partial \delta P}{\partial x_{i}} d t+d M_{i},
$$

where the pressure increments $\delta P$ are computed to impose that $\operatorname{div}\left(\left\langle\mathbf{U}_{p}\right\rangle\right)=0$. A classical numerical derivation based on the fractional time-step approach yields that $\delta P$ is the solution of the Poisson equation

$$
\frac{\Delta t}{\rho_{f}} \Delta(\delta P)=\operatorname{div}\left(\left\langle\widetilde{\mathbf{U}}_{p}\right\rangle\right) .
$$

As such, this correction on particle velocities is equivalent to using a Poisson solver twice. As such, better accuracy and consistency is only obtained at the cost of doing this operation.

In order to test the importance of the correction for particle velocities, an approximate method is used where particle velocities in a each cell are shifted so that the mean velocity field from the Lagrangian solver is equal to the one computed by the Eulerian solver. This is translated by the following correction: for each particle labeled $[k]$, with $k=1, \ldots, N_{\mathbf{x}_{m}}$ where $N_{\mathbf{x}_{m}}$ is the number of particles located in the cell (here $m$ refers to cell number and $\mathbf{x}_{m}$ to its center), its instantaneous velocity is modified as

$$
U_{p, i}^{[k]}(t)=\widetilde{U}_{p, i}^{[k]}(t)-\left(\left\langle\widetilde{U}_{p, i}\right\rangle\left(t, \mathbf{x}_{m}\right)-\left\langle U_{f, i}\right\rangle\left(t, \mathbf{x}_{m}\right)\right)
$$

to enforce that $\left\langle U_{p, i}\right\rangle(t, \mathbf{x})=\left\langle U_{f, i}\right\rangle(t, \mathbf{x})$ and retrieve a divergence-free mean velocity field. 


\subsubsection{Numerical implementation}

The calculations were performed using Code_Saturne, an open-source CFD code developed at Electricité de France Recherche \& Développement (EDF R\&D). Details on the numerical methods implemented in the code can be found in Archambeau et al. (2004) for the Eulerian solver and in Peirano et al. (2006) for the Lagrangian one.

Since the selected numerical cases correspond to steady-state flows, hybrid Eulerian/Lagrangian simulations are performed sequentially:

- first, the Eulerian solver is run and, at convergence, calculations are stopped and the mean velocity, pressure, and turbulence fields are frozen. These simulations are obtained with the second-order Rotta turbulence model (with $C_{R}=4.15$ );

- second, particles are introduced and tracked within the pre-calculated flow fields. The SLM is retained to model fluid particle velocity evolutions (with $\left.C_{0}=2.1\right)$

\section{Numerical Assessment}

In this section, we illustrate the points addressed in Sect. 2 with numerical applications. To this end, three cases are studied:

- a mixing layer (free shear-flow);

- an infinite channel flow (simple wall-bounded flow);

- a flow around an obstacle in a boundary layer (complex wall-bounded flow).

These three cases allow testing of theoretical and numerical formulations in situations where different effects come into play. The mixing layer case is a nonhomogeneous turbulent flow, with a laminar/turbulent transition at the edges of the mixing layer but without flow reversal or wall effects. On the other hand, the infinite channel flow is a simple one-dimensional but non-homogeneous flow. Finally, the case of the flow around an obstacle located on a wall in a atmospheric boundary layer gathers all difficulties: it is a non-homogeneous turbulent flow, involving wall effects and complex flow recirculation patterns that make it a stringent test case to assess the respect of the well-mixed criterion and consistency requirements.

\subsection{The Mixing Layer Case}

\subsubsection{Case and Simulation Description}

Case description Mixing layers correspond to turbulent flows formed between two uniform, nearly parallel streams of different flow speeds $U_{\max }$ and $U_{\text {min }}$. Figure 2a displays the typical sketch of a mixing layer created by the interaction between two streams with different flow speeds. 


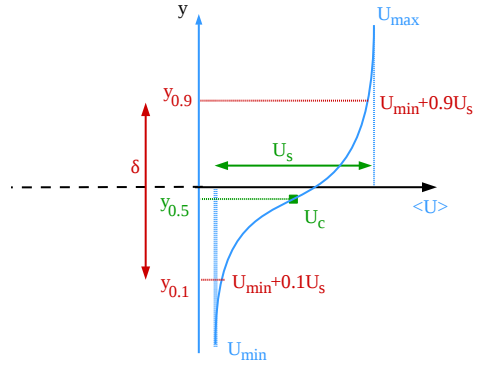

(a)

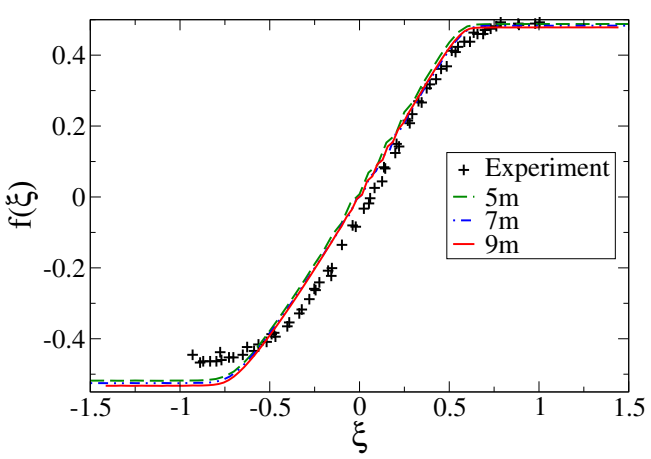

(b)

Fig. 2 Sketch and profile of a mixing layer. (a) Sketch of a $2 \mathrm{D}$ mixing layer (with the characteristic parameters used and the velocity profile). (b) Profile of the scaled mean longitudinal velocity across the mixing layer: experiments (symbols) and simulations (lines) at various distances from the inlet

Simulation set-up Numerical simulations of the fluid flow are performed within a rectangular domain, whose dimensions are $10 \mathrm{~m} \times 0.8 \mathrm{~m} \times 0.004 \mathrm{~m}$, with two different inlet velocities. The grid is composed of $100 \times 100 \times 1$ rectangular uniform cells. The boundary conditions are as follows: the inlet conditions on the left-hand side (l.h.s.) of Fig. 2a are divided in two regions, each with a uniform velocity profile $\left(U=2 \mathrm{~m} \mathrm{~s}^{-1}\right.$ for $y<0.4 \mathrm{~m}$ and $U=3 \mathrm{~m} \mathrm{~s}^{-1}$ for $y>0.4 \mathrm{~m}$ ); the upper and lower boundaries are symmetry (zero-flux) conditions while an outlet condition is applied on the right-hand side of Fig. 2a. Thus, the parameters used for dimensionless quantities are equal to: $U_{\min }=2 \mathrm{~m} \mathrm{~s}^{-1}$ and $U_{s}=1 \mathrm{~m} \mathrm{~s}^{-1}$.

Once a stationary regime is reached for the fluid flow, fluid particles are then injected until a steady-state regime is reached (i.e. with a statisticallyconstant number of particles. In numerical simulations, each fluid particle represents an equal amount of fluid mass and, consequently, the number of particles being injected through the two inlet subsections corresponding to the two different streams must be so that the inlet mass flow rates are respected. Then, given the velocities imposed at the inlet for the two streams $\left(2 \mathrm{~m} \mathrm{~s}^{-1}\right.$ for $y<0.4 \mathrm{~m}$ and $3 \mathrm{~m} \mathrm{~s}^{-1}$ for $y>0.4 \mathrm{~m}$ ), it was chosen to inject 80 particles during each timestep through the low-speed stream inlet (for $y<0.4 \mathrm{~m}$ ), which means that 120 particles were injected through the high-speed stream one (for $y>0.4 \mathrm{~m}$ ). When a steady-state was reached, the domain contained a number of particles of the order of $N=80000$, i.e. about 8 particles per cell. In the steady-state regime, Monte Carlo estimates were averaged over time, using an ergodic assumption, to reduce statistical noise to low levels: here, results have been averaged over 2000 timesteps, meaning that 16000 particles per cell were used to calculate statistics. 


\subsubsection{Numerical results}

Self-similarity of the flow Numerous studies have shown that the flow becomes self-similar after an initial adjustment period and that the flow can be written simply in terms of a scaled velocity (more details can be found in Champagne et al. (1976); Pope (2000)) as follows

$$
f(\xi)=\frac{\langle U\rangle-U_{c}}{U_{s}},
$$

which depends only on the scaled cross-stream location (self-similar flow)

$$
\xi=\frac{y-\bar{y}(x)}{\delta(x)}
$$

where $U_{c}=\frac{1}{2}\left(U_{\max }+U_{\min }\right)$ is the characteristic convection velocity, $U_{s}=$ $\left(U_{\max }-U_{\min }\right)$ is the characteristic velocity difference, $\delta(x)=y_{0.9}(x)-y_{0.1}(x)$ is the characteristic width of the flow (defined as the extent of the region where the fluid velocity ranges from $U_{\min }+0.1 U_{s}$ to $U_{\text {min }}+0.9 U_{s}$ ) (see Fig. 2a). This self-similarity is well captured by the numerical simulations as appears from the scaled longitudinal mean velocity profiles (see Fig. 2b). Similarly, the Reynolds stresses have been shown to be self-similar when scaled by $U_{s}^{2}$ (not shown here).

Well-mixed criterion Since the objective of the simulation is to assess whether the well-mixed criterion is respected, we consider the normalized particle concentration and check that it remains constant in the simulation domain (particles should not accumulate in nor be removed from regions). The relevant statistical measure is the normalized particle number, or particle concentration, obtained by dividing the actual number of particles in any cell by the mean one.

This normalized particle concentration is displayed in Fig. 3. The horizontal concentration profile taken at mid height $(y=0.4 \mathrm{~m})$, that is at the same height as the splitter plate, is plotted in Fig. 3a. A depletion zone can be seen near the inlet (roughly between the inlet and $x=1 \mathrm{~m}$, whose value depends on the choice of initial velocities and mesh sizes). This depletion region corresponds to the distance needed for the particles to correctly mix in the domain: particles are indeed injected every timestep exactly on the boundary face (and not within the whole boundary cell). This simple injection procedure has been chosen to show that a properly-formulated Lagrangian stochastic model can mix particles and drive the distribution of particle positions to a uniformlydistributed one. Once correctly mixed, the particles remain homogeneously distributed.

Role of correction on particle velocities The influence of the particle velocity correction (see Eq. 6) can be estimated by considering the mixing layer case. When calculations are run without this velocity correction, it is seen from 


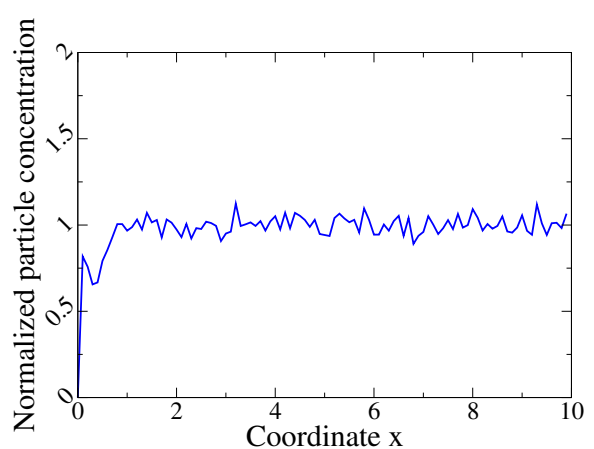

(a) Horizontal profile $(y=0.4 \mathrm{~m})$

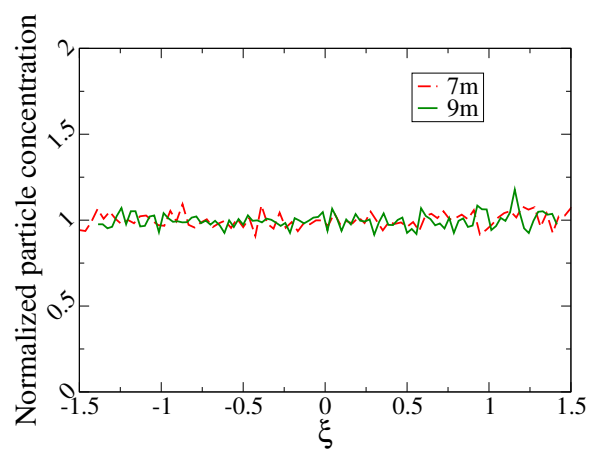

(b) Vertical profiles

Fig. 3 Profiles of the particle normalized concentration in the plane mixing layer: (a) sampled longitudinally along the middle of the layer; (b) sampled vertically at various distances from the inlet

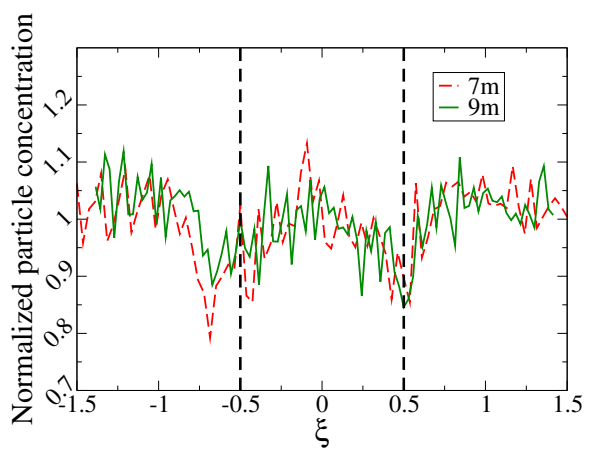

(a) Without particle velocity correction

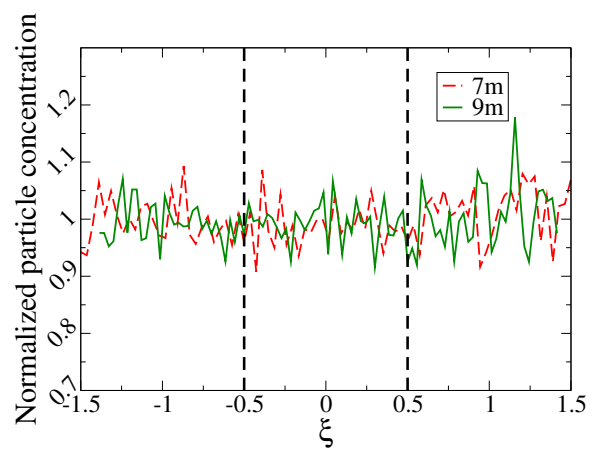

(b) With particle velocity correction

Fig. 4 Profiles of the particle normalized concentration in the mixing layer at various distances from the inlet: comparison between the formulations without and with particle velocity correction (cf. Eq. 6). The dashed lines correspond to the laminar-turbulent transitions

Fig. 4a that the normalized-concentration field is only approximately uniform, with deviations from unity apparent at the edges of the mixing layer. This is related to the fact that the external fluid is entrained to the mixing-layer core, where the flow is turbulent, resulting in the external intermittency of the flow. However, when this velocity correction is applied, a better result is obtained, as revealed by the profiles in Fig. 4b. This confirms that the particle velocity correction scheme described in Sect. 2.2.3 is required to obtain more accurate results.

Consistency of turbulence models Scaled Reynolds stresses are extracted from the Lagrangian calculation and compared to the ones from the Rotta model used in the CFD code. Both predictions of the scaled non-zero second-order fluid velocity moments are shown in Fig. 5. It can be seen that the Reynolds stresses decrease as expected at the edges of the turbulent region where a tur- 


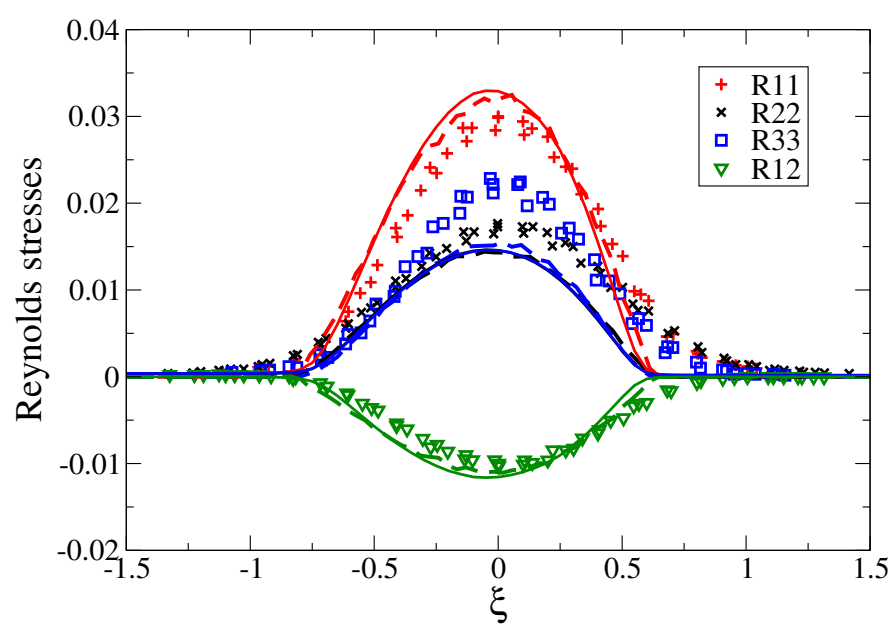

Fig. 5 Profiles of the scaled Reynolds stresses in the self-similar regime (obtained here at $7 \mathrm{~m}$ ) for the mixing layer case provided by experimental data (points), the Eulerian solver (continuous lines) and the Lagrangian one (dashed lines) with the consistent hybrid Rotta model/SLM formulation. Both approaches yield nearly-identical predictions for the same second-order velocity moments.

bulent/laminar transition is observed. More importantly for our present concern, it is clear that numerical results obtained with the Lagrangian model are in very good agreement with those obtained with the Eulerian model, indicating that consistency is well achieved with the hybrid SLM-Rotta calculation. Besides, it can be seen that the numerical results obtained compare fairly well to the experimental observations available for the mixing layer case (see also Minier and Pozorski (1995) for detailed comparison between numerical and experimental results in the mixing layer case).

\subsection{The Infinite Channel Flow}

\subsubsection{Case and Simulation Description}

Case description The second test case corresponds to an infinite half-channel flow, which is a simple one-dimensional but non-homogeneous flow. This case is representative of wall-bounded flows, i.e. that are affected by the wallboundary conditions.

Simulation set-up Simulations are performed in a vertical slice of 20 cells, with dimensions of $0.05 \mathrm{~m} \times 0.05 \mathrm{~m} \times 0.05 \mathrm{~m}$. Since this is basically a onedimensional flow where statistics depend only on the wall-normal coordinate, the flow is simply generated by applying a periodicity condition between the upstream and downstream faces and by imposing in the longitudinal direction a momentum source term that corresponds to the mean pressure gradient (or 
pressure loss) $d\left\langle P_{f}\right\rangle / d x=-\rho_{f} u_{*}^{2} / H$, where $\rho_{f}=1.18 \mathrm{~kg} \mathrm{~m}^{-3}$ is the density of air, $H=1 \mathrm{~m}$ the half-height of the channel, and $u_{*}=0.05 \mathrm{~m} \mathrm{~s}^{-1}$ the chosen value of the friction velocity at the wall.

Then, $N=200000$ particles uniformly distributed over the domain are injected. Periodic boundary conditions are applied in the upstream and downstream faces, wall-boundary conditions are applied at the wall while symmetry conditions are applied in the other directions. As a result, there is neither inlet nor outlet for particles. Particles are then tracked during a time-lapse long enough that results truly reflect the governing stochastic model rather than the memory of the initial conditions.

\subsubsection{Numerical results}

Well-mixed criterion The purpose of this simulation is to check that a steady state is reached, where particle concentration remains uniform across the whole channel. As in the mixing layer case, we only consider normalized concentrations. The average number of particles in a cell is around 10000 , which is high-enough for statistics to be obtained directly through Monte Carlo estimations without using any time-averaging. Incidentally, this allows assessment of whether statistics remain stationary in time.

Figure 6 shows a scatter plot of particle positions in the domain at the end of the calculation, along with the corresponding concentration vertical profile derived from it. It can be seen that the concentration is constant over the whole height of the channel, indicating that a truly uniform particle distribution is obtained. For a more quantitative analysis, the vertical profiles of the normalized concentration $c / c_{0}$ (where $c_{0}$ is the mean concentration in the whole domain) derived at different timesteps in the calculation are displayed in Fig. 7a. These profiles show that uniformity across the channel is maintained in time. Besides, it can be seen that the normalized concentration profiles oscillate with some noise around 1 and it is interesting to analyze the nature of these fluctuations. For that purpose, we consider the global spatial error defined as

$$
\frac{\sigma_{c}}{c_{0}}=\frac{1}{c_{0}} \sqrt{\frac{\sum_{j=1}^{N_{\text {cells }}}\left(c\left(\mathbf{x}_{j}\right)-c_{0}\right)^{2}}{N_{\text {cells }}}},
$$

where $N_{\text {cells }}=20$ is the number of cells and $\mathbf{x}_{j}$ denotes the position of the center of the computational cells. Particle concentration is uniform in the domain if and only if the global spatial error $\sigma_{c} / c_{0}$ equals zero. In the present case, it is found to be $0.626 \%$ at the end of the calculation. However, since we are dealing with Monte Carlo estimates, statistical error needs to be accounted for. From the Central limit theorem, this error writes

$$
\left(\frac{\sigma_{c}}{c_{0}}\right)_{\text {Monte Carlo }}=\sqrt{\frac{N_{\text {cells }}}{N}},
$$

where $N$ is the number of particles in the domain. With present values, this Monte Carlo error is therefore equal to $1 \%$. Finally, the local error in each 


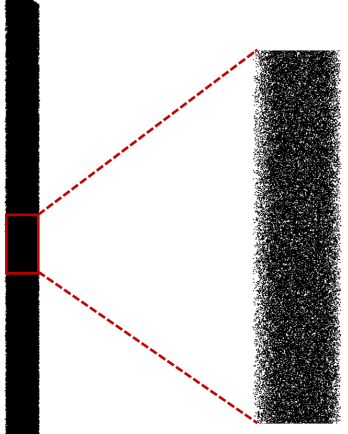

(a)

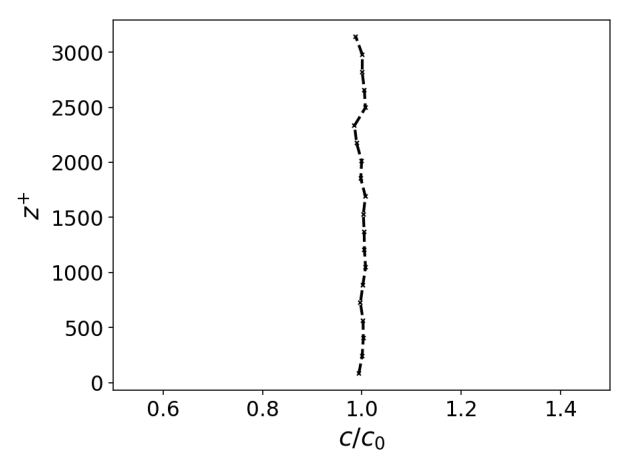

(b)

Fig. 6 Particle repartition at the end of the calculation (a) and the corresponding profile of the particle concentration (b), showing the respect of the well-mixed criterion

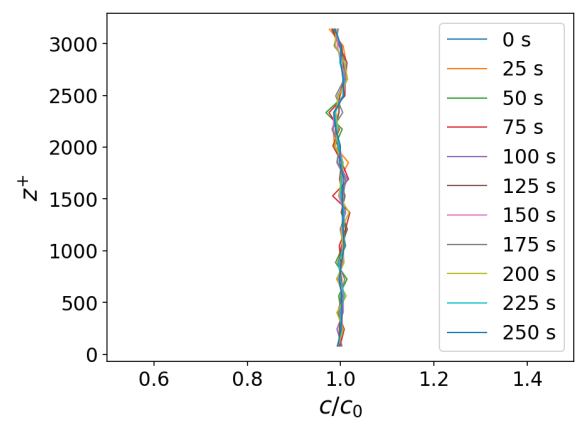

(a) With the mean pressure gradient term

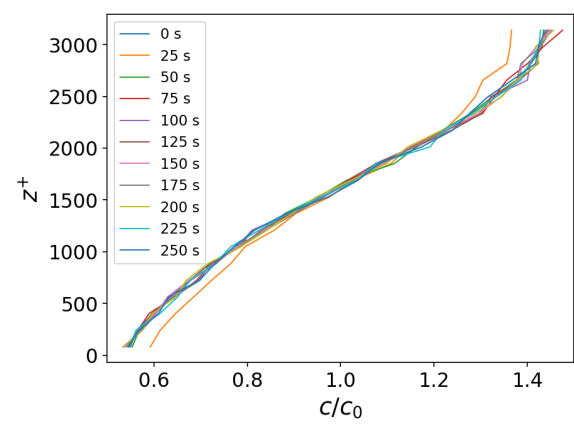

(b) Without the mean pressure gradient term

Fig. 7 Vertical profiles of the normalized particle concentration $\left(c / c_{0}\right)$ at different timesteps: (a) with the mean pressure gradient term; (b) without it

cell (defined as $\left(c-c_{0}\right) / c_{0}$ and evaluated after the solution has reached a steady state and $c$ a constant value) is found to remain within $-1.48 \%$ and $0.074 \%$, which confirms that a uniform particle distribution is achieved across the height of the channel.

Role of pressure gradient term As revealed by the normalized concentration profiles shown in Fig. 7b, particles tend to accumulate at the top of the domain when the mean pressure gradient term is removed from the Langevin model (see Sect. 2.1). In the case of the infinite channel flow, it is easy to understand why: in the absence of the mean pressure-gradient term, particles are driven towards low-energy zones. Indeed, the classical expression of the mean-momentum balance equation in the wall-normal direction gives that

$$
\frac{1}{\rho_{f}} \frac{d\left\langle P_{f}\right\rangle}{d z}+\frac{d R_{33}}{d z}=0
$$




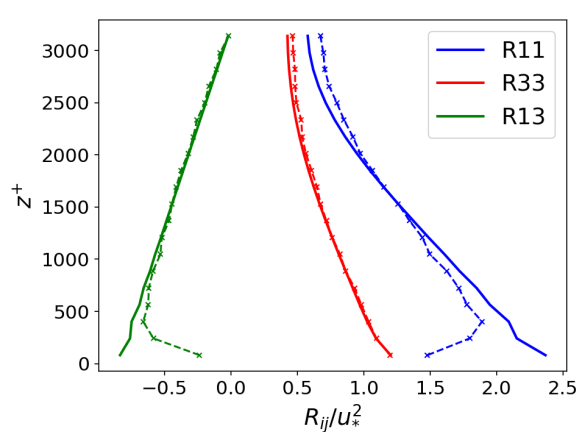

(a) Specular rebound

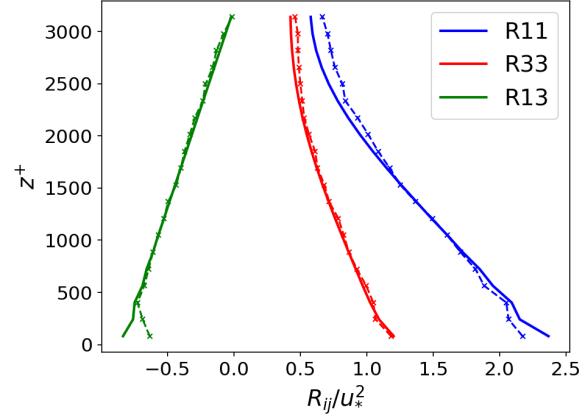

(b) Wall-boundary condition Eq. 2

Fig. 8 Reynolds stress vertical profiles for the case of the infinite plane channel: comparison between two versions of wall-boundary conditions with the consistent hybrid Rotta/SLM formulation. In both versions, consistency is assessed by checking the similarity of the predictions from the Eulerian solver (continuous lines) and the Lagrangian one (dashed crosses)

showing that removing the pressure-gradient term is equivalent to a spurious particle driving force along the direction of $d R_{33} / d z$. In that case, significant errors (reaching values up to $30.8 \%$ ) are introduced, indicating that the general formulation without the mean pressure-gradient is deeply flawed.

Consistency of boundary conditions The profiles of the non-zero Reynolds stresses are displayed in Fig. 8. The effect of using consistent wall-boundary conditions can clearly be observed: the agreement between the Reynolds-stress values extracted from the Eulerian and Lagrangian solvers is much better with the current boundary conditions (see Sect. 2.2.2) than when specular rebound is used. In particular, the improvement is notable for $R_{11}$ and $R_{13}$ with the difference between Eulerian and Lagrangian values being reduced by half. It can be seen that $R_{33}$ is not affected, which makes sense since the normal velocity component remains unchanged (see Eq. 2). The difference between the resulting profiles of the shear stress $R_{13}$ is noteworthy since this is the key element of near-wall turbulent boundary layers (also called constant-stress layers) when the viscous sublayer is bridged: applying a specular rebound yields a zero-flux condition for the mean longitudinal velocity component and a zero shear stress completely at odd with classical laws of wall-boundary layers at high Reynolds-number turbulent flows.

In practical numerical formulations of wall-boundary conditions, specific discretizations are sometimes used. This is the case for the present implementation of wall-conditions in the CFD code Code_Saturne, which can explain remaining differences in the numerical outcomes. Further work needs to be done to ensure a full correspondence down to the actual numerical formulations. Nevertheless, it can be concluded that wall-boundary conditions are necessary in Lagrangian stochastic formulations and must be implemented consistently with the ones applied in the Eulerian solver when a hybrid formulation is used. 


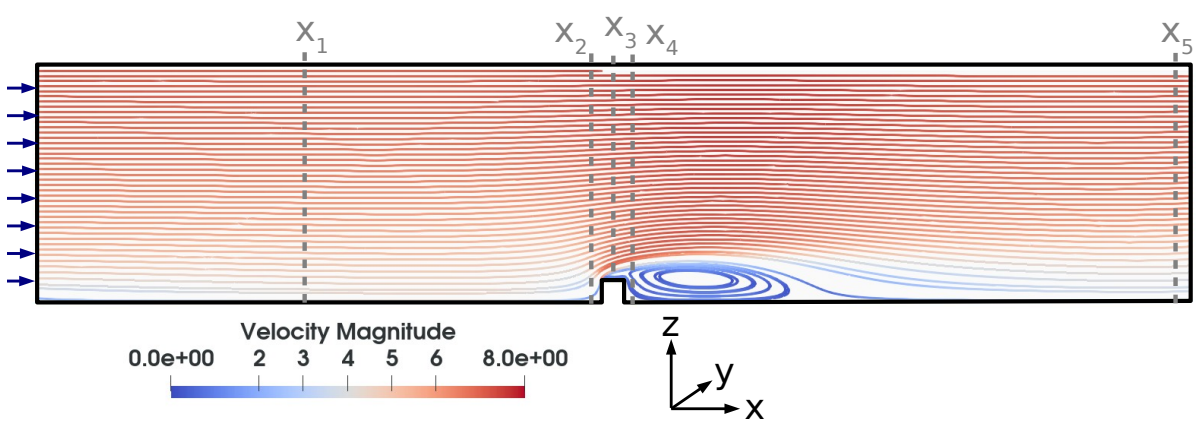

Fig. 9 Geometry, streamlines and velocity field of a flow around an obstacle within a boundary layer. It reveals the complexity of the flow patterns around the obstacle with a double recirculation on top and downstream. The figure also displays the vertical positions at which profiles are measured

\subsection{Obstacle within a Boundary Layer}

\subsubsection{Case and Simulation Description}

Case description The third test case studied in this work corresponds to a flow around a square obstacle placed on the bottom wall inside a boundary layer. The spanwise dimension of the square obstacle is large enough to be regarded as infinite with respect to other characteristic lengths and the mean flow is therefore a 2D flow. This test case is representative of flows over large obstacles that are usually addressed in atmospheric dispersion studies (as for instance the flow over a building). Yet, as the other cases described earlier in this paper, we are considering a simplified situation. In that sense, these simulations correspond to a 'verification test case', meaning that we are checking that the formulation for the model of the fluid flow and for the model of particle dynamics are consistent, and not to a 'validation case' (where results are compared to experimental data).

Simulation set-up The geometry used to perform such simulations is sketched in Fig. 9: the domain corresponds to a box with sizes equal to $5 \mathrm{~m} \times 0.1 \mathrm{~m} \times$ $1 \mathrm{~m}$. The mesh is simply made up by 798400 cubic cells. The case considered is a rough boundary layer consisting of a smooth surface with a building (similar to what could be simulated in a wind tunnel with a smooth test section). The flow is initialized with a uniform flow (given also by the same upstream profile). The boundary conditions are thus as follows: the inlet condition reproduces the flow features typical of rough boundary layers (with a roughness height $z_{0}$ ), as detailed in Table 3 (note that with the present dimensions, the mean longitudinal velocity component ranges from a near-zero value close to the wall to about $10 \mathrm{~m} \mathrm{~s}^{-1}$ at the upper boundary plane); a symmetry condition is applied on the upper boundary while a smooth wall condition is applied to the bottom boundaries. 
Table 3 Inlet dynamical fields for the case of the obstacle within a boundary layer

\begin{tabular}{ll}
\hline Field & Value or expression \\
\hline$\left\langle U_{f, x}\right\rangle\left(\mathrm{m} \mathrm{s}^{-1}\right)$ & $\frac{u_{*}}{\kappa} \ln \left(\frac{z+z_{0}}{z_{0}}\right)$ \\
$\left\langle U_{f, y}\right\rangle\left(\mathrm{m} \mathrm{s}^{-1}\right)$ & 0 \\
$\left\langle U_{f, z}\right\rangle\left(\mathrm{m} \mathrm{s}^{-1}\right)$ & 0 \\
$u_{*}\left(\mathrm{~m} \mathrm{~s}^{-1}\right)$ & 0.5 \\
$k\left(\mathrm{~m}^{2} \mathrm{~s}^{-2}\right)$ & $\frac{u_{*}}{\sqrt{C_{\mu}}}, C_{\mu}=0.09$ \\
& $\frac{u_{*}^{3}}{\kappa\left(z+z_{0}\right)}, \kappa=0.42, z_{0}=0.0025 \mathrm{~m}$ \\
$\epsilon_{f}\left(\mathrm{~m}^{2} \mathrm{~s}^{-3}\right)$ & $(2 / 3) k$ \\
$\left\langle U_{f, x}^{\prime 2}\right\rangle\left(\mathrm{m}^{2} \mathrm{~s}^{-2}\right)$ & $(2 / 3) k$ \\
$\left\langle U_{f, y}^{\prime 2}\right\rangle\left(\mathrm{m}^{2} \mathrm{~s}^{-2}\right)$ & $(2 / 3) k$ \\
$\left\langle U_{f, z}^{\prime 2}\right\rangle\left(\mathrm{m}^{2} \mathrm{~s}^{-2}\right)$ & 0 \\
$\left\langle U_{f, x}^{\prime} U_{f, y}^{\prime}\right\rangle\left(\mathrm{m}^{2} \mathrm{~s}^{-2}\right)$ & $-u_{*}^{2}$ \\
$\left\langle U_{f, x}^{\prime} U_{f, z}^{\prime}\right\rangle\left(\mathrm{m}^{2} \mathrm{~s}^{-2}\right)$ & 0 \\
$\left\langle U_{f, y}^{\prime} U_{f, z}^{\prime}\right\rangle\left(\mathrm{m}^{2} \mathrm{~s}^{-2}\right)$ & 0 \\
\hline
\end{tabular}

In order to test different initialization procedures, particle simulations were not run starting from an initially void-of-particle domain as for the mixing layer case but by initializing a large number of particles $\left(N_{i n i}=2928000\right)$ in the domain with a uniform position distribution to respect incompressibility. In each cell, particle velocities were initialized by sampling in a Gaussian distribution determined by the local value of the mean fluid velocity and second-order moments (the local value of the Reynolds-stress tensor). Unlike the infinite channel flow situation, this is a case with exit and inlet sections but with no periodic conditions. Therefore, during each timestep, a number of fluid particles $\left(N_{\text {inlet }}=352\right)$ are injected through the inlet section, according to the profile of the inlet mass flow rate. More precisely, for each cell $k$ corresponding to height $z_{k}: N_{k}=\left(q_{k} / Q\right) N_{\text {inlet }}$, where $N_{k}$ is the number of particles in cell $k$ and $Q$ the global fluid flow rate at the inlet. Also, $q_{k}=\left\langle U_{f}\right\rangle_{k} S_{k}$, where $\left\langle U_{f}\right\rangle_{k}$ is the mean fluid velocity in cell $k$ and $S_{k}$ is the surface of the boundary face related to cell $k$. Since the simulation is initialized with a uniform flow, we run the simulation until a stationary state is reached. This also ensures that particles lose memory of the initial conditions. Results plotted in the later figures are thus not influenced by the initial conditions of the simulation. Nevertheless, this initialization is useful for avoiding the time-consuming explicit simulation of the transient period where particles have to fill the whole domain and acts as an accelerator of convergence. When a steady-state is reached, the typical number of particles present in the domain is in the order of $N=3000000$, i.e. about 4 particles per cell. Therefore, to extract reliable statistics, a time-averaging procedure is applied as in the mixing layer case. To ensure results independent of the number of samples, time averaging is performed over 200000 timesteps (meaning that, on average, statistics are estimated with around 800000 particles in each cell). 
Note that for this case, no typical scales can be used in the whole domain, as $u_{*}$ is not a constant and there is no self-similarity. Therefore, apart from the mean concentrations, plots are displayed in terms of dimensional variables.

\subsubsection{Numerical Results}

Fluid flow Fluid phase simulations are run until a statistically steady-state is reached. As expected, a strongly non-homogeneous flow is induced by the impact of the incoming stream on the obstacle, with high levels of kinetic energy in the high-shear region appearing downstream of the obstacle. A large recirculation zone is formed downstream of the obstacle but a zoom of the region just above the obstacle indicates that another recirculation is created between the top wall of the obstacle and the main stream flowing above. This case thus represents a very complex flow structure, involving wall effects, recirculating and high-shear regions. It is thus a challenging situation for the simulation of particle dynamics and, consequently, a severe test to assess the respect of the well-mixed criterion.

Well-mixed condition As for the mixing layer and infinite channel cases, the variable of interest is the normalized particle concentration. To assess the accuracy of the current approach, the normalized particle concentration is sampled along five vertical profiles at various downstream distances from the inlet section, as shown in Fig. 9: a first profile is taken upstream of the obstacle (sufficiently far from the inlet to have a fully-developed channel flow), three profiles just before, above, and just downstream of the obstacle and a last one at the end of the domain downstream of the obstacle. The five selected profiles of the normalized concentration are shown in Fig. 10a: these profiles confirm that a relatively well-mixed condition is obtained as all the different profiles remain fairly constant with some fluctuations inherent to particle stochastic simulations. The results close to the boundary are not perfect though (particles are less present). This may be due to the complex flow structure, which is not properly captured with the current log-normal boundary conditions used. Improvements of these boundary conditions to better fit this case are left out of the present paper but will be investigated in the near future. Given the challenging flow structure on the top of the obstacle, the fact that the normalized particle concentration remains relatively spatially and temporally uniform is a noteworthy result. The global spatial error, defined in Eq. 9, is $5.06 \%$ at the end of the calculation and is therefore satisfactorily low.

However, when the mean pressure gradient term is not taken into account in the SLM (see Sect. 2.2.3), a completely different picture emerges with marked deviations of particle concentration from uniformity, with strong accumulation upstream of the obstacle (where fluid streamline curvatures are important) and zones of depletion downstream of the obstacle (in the recirculation region). When profiles of the normalized concentration are extracted at the same five locations, it appears from the plots in Fig. 10b that severe errors are present, with some values reaching as high as four times the correct one. In that case, 


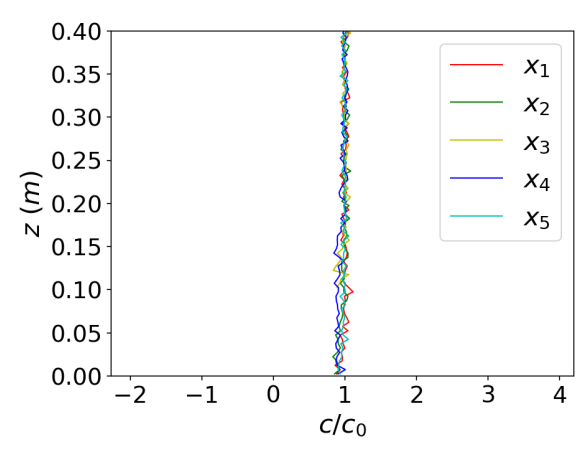

(a) With the mean pressure gradient term

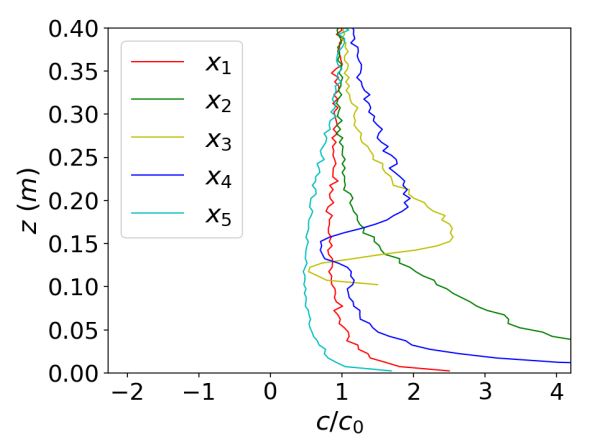

(b) Without the mean pressure gradient term

Fig. 10 Vertical profiles of the particle normalized concentration at the five sections indicated in Fig. 9 for the case of the flow around an obstacle within a boundary layer. Comparison between the formulations with and without the mean pressure gradient in the SLM. In Fig. 10b, the $x_{2}$ and $x_{4}$ profiles are not entirely visible because, for these profiles, the ratio $c / c_{0}$ reached values up to over 20 (showing a deep violation of the well-mixed criterion). Therefore, for the sake of readability and keeping the same scales for both figures, the $x$-scale has been contracted for Fig. $10 \mathrm{~b}$

the global error is measured as high as $52.8 \%$. This is clearly an unacceptable level of error, which invalidates such formulations of dispersion models.

Correction of particle positions The correction on particle velocities introduces a somewhat asymmetric treatment of particle positions and velocities, since the evolution equations for $\left(\mathbf{X}_{p}, \mathbf{U}_{p}\right)$ can be written as:

$$
\begin{aligned}
d \widetilde{X}_{p, i} & =\widetilde{U}_{p, i} d t \\
d U_{p, i} & =d \widetilde{U}_{p, i}-\frac{1}{\rho_{f}} \frac{\partial \delta P}{\partial x_{i}} d t
\end{aligned}
$$

where the same notation $\widetilde{\mathbf{X}}_{p}$ for particle positions is used to indicate that this is a first estimation. Eq. 12a shows that particle positions are influenced by the velocity correction but only in a sequential manner: once particle velocities are corrected, particle positions are modified at the following timestep. This loose coupling can be sufficient in some cases, such as the mixing layer and infinite channel flow cases, but can be limited for more complex flow patterns with flow reversals and recirculation. This is the situation met with the flow around the obstacle in the boundary layer. For this reason, tighter coupling is needed between particle positions and velocities.

In stand-alone PDF approaches (see Minier and Pozorski (1999); Bernardin et al. (2009); Chauvin et al. (2010)), as well as in Smoothed Particle Hydrodynamics (SPH) simulations of incompressible flows (Szewc et al. 2012), this is done by introducing a specific correction on particle positions, so that the 
complete system can be represented by

$$
\begin{aligned}
& d X_{p, i}=\widetilde{U}_{p, i} d t+\delta X_{p, i}, \\
& d U_{p, i}=d \widetilde{U}_{p, i}-\frac{1}{\rho_{f}} \frac{\partial \delta P}{\partial x_{i}} d t,
\end{aligned}
$$

where in Eq. 13a the correction term $\delta X_{p, i}$, accounts for the integrated effect (within each timestep) of the one acting on particle velocities in Eq. 13b. This particle correction term is derived by stating that a uniform particle concentration field should be obtained. Expressing $\delta X_{p, i}$ as the integrated effect of a potential correcting field $\delta \phi$, i.e. with $\delta X_{p, i}=\partial(\delta \phi) / \partial x_{i} \Delta t$, leads to the formulation of a second Poisson equation whose solution is this correcting potential $\delta \phi$. Further details on this numerical implementation with corrections on particle velocities and positions can be found in Minier and Pozorski (1999).

As for particle velocities, a short-cut method was used to assess whether such a complete treatment is needed. To that effect, particle positions are simply modified by adding a Brownian-like term to the particle position equation, so that we have now

$$
\begin{aligned}
d X_{p, i} & =\widetilde{U}_{p, i} d t+\alpha d W_{i}^{\prime}, \\
d U_{p, i} & =d \widetilde{U}_{p, i}-\frac{1}{\rho_{f}} \frac{\partial \delta P}{\partial x_{i}} d t
\end{aligned}
$$

where $\alpha$ is a small parameter and $\mathbf{W}^{\prime}$ a set of Wiener processes independent of the ones used in the Langevin model for particle velocities. This corresponds to applying a simple shifting term to particle positions, or a Laplacian term in the (Fokker-Planck type of) PDE governing the evolution of the particle concentration field.

In the case of the flow around the obstacle, this version was used to obtain the numerical results presented in Sect. 3.3. In the simulations, $\alpha \approx 0.12$ $\mathrm{m} \mathrm{s}^{-3 / 2}$, but $\alpha$ nevertheless remains an adjustable parameter in the present context. The effects of this particle correction scheme can be seen by comparing results on the particle concentration field with and without that term (cf. Fig. 11). Clearly, improved predictions are obtained when the correction term is applied. Given the simplified version used in Eq. 14a, this indicates that the complete formulation in terms of $\delta \phi$ as the solution of the corresponding Poisson equation needs to be introduced. This issue will be investigated separately in a future study.

Consistency of turbulence models We now consider the case of the flow around an obstacle in a boundary layer, which is more complex since it involves wall effects and flow recirculation. Following the analysis in Sect. 3.3, three zones (upstream, over, and downstream of the obstacle) are selected where vertical profiles are extracted. This is shown in Fig. 12a, c, e for the consistent hybrid Rotta-SLM formulation. It can be seen that the profiles computed through the Eulerian and Lagrangian approaches are quite similar, especially $R_{33}$ where the identity is almost perfect. There are, however, small differences for $R_{11}$ 


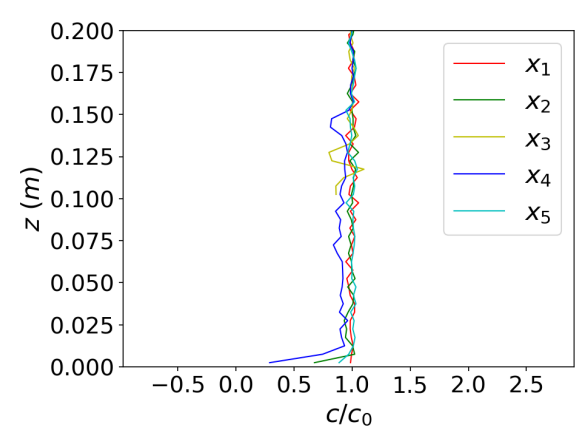

(a) Without particle position correction

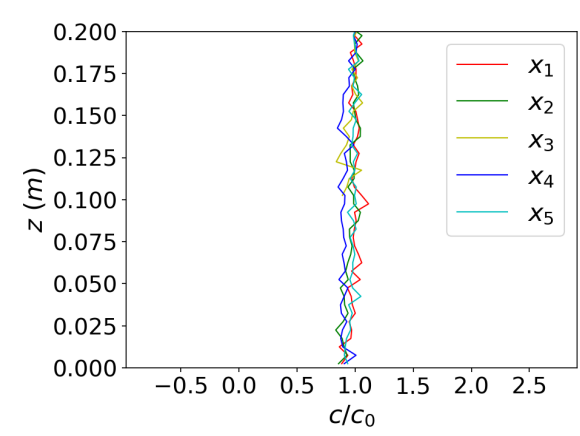

(b) With particle position correction

Fig. 11 Vertical profiles of the particle normalized concentration at the five sections shown in Fig. 9 for the case of the flow around the obstacle within a boundary layer: comparison between the formulations with and without particle position correction, Eq. 14a. The particle velocity correction, Eq. 6, is applied in both formulations

values, which is the longitudinal fluid kinetic energy. At that stage, it must be recalled that, from a numerical point of view, consistency between Eulerian and Lagrangian approaches cannot be perfectly enforced since the triple moment has to be modeled in the Eulerian formulation (usually through a Daly-Harlow closure) while it is treated without approximation by particlebased approaches. Over the obstacle, second-order moments are in a very good agreement, which is a noteworthy result given the possible difficulties caused by the strong recirculating flow patterns present in the region. In spite of small differences that still require further analysis, it can nevertheless be concluded that a satisfactory agreement between both Eulerian and Lagrangian results are obtained for these duplicate fields, which brings support for the overall hybrid formulation.

To further illustrate the importance of consistent formulations, another simulation was performed using the $k-\epsilon$ turbulence model for the Eulerian calculation. Results are shown in Fig. 12b, d, f, which displays the turbulence kinetic energy vertical profiles at the same locations as the ones used with the $R_{i j}-\epsilon$ Rotta model for the Eulerian solver (Fig. 12a, c, e). It is obvious that significant discrepancies are observed between predictions of the same physical quantity, proving that this hybrid formulation is inconsistent and, therefore, invalid for reliable predictions.

\section{Conclusions and Perspectives}

The purpose of the present study is to help the development and use of Lagrangian stochastic models for atmospheric dispersion applications by clarifying, through theoretical considerations and a range of numerical situations, the issues of the well-mixed condition and consistencies between duplicate fields in hybrid Eulerian/Lagrangian simulations. 


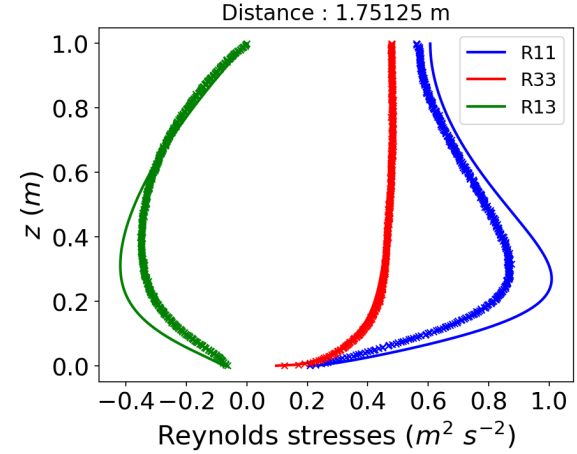

(a) $R_{i j}-\epsilon$ model, upstream of the obstacle

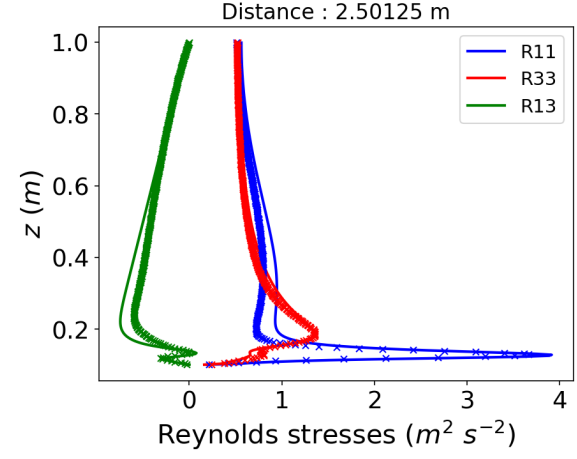

(c) $R_{i j}-\epsilon$ model, over the obstacle

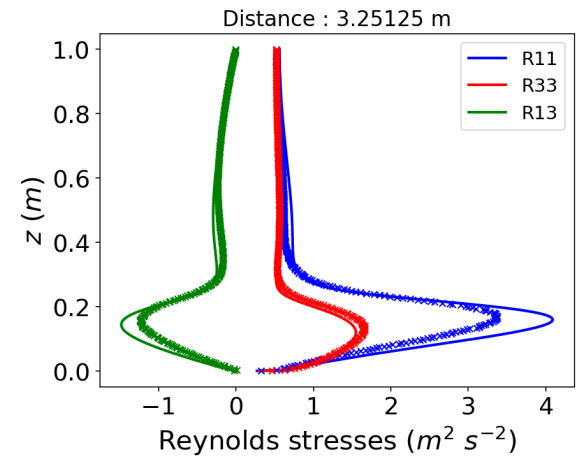

(e) $R_{i j}-\epsilon$ model, downstream of the obstacle

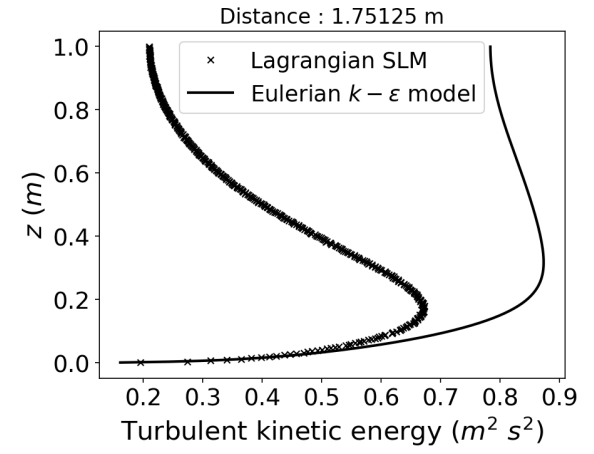

(b) $k-\epsilon$ model, upstream of the obstacle

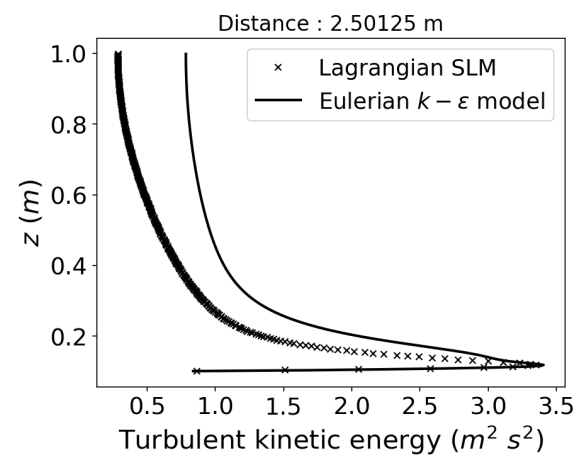

(d) $k-\epsilon$ model, over the obstacle

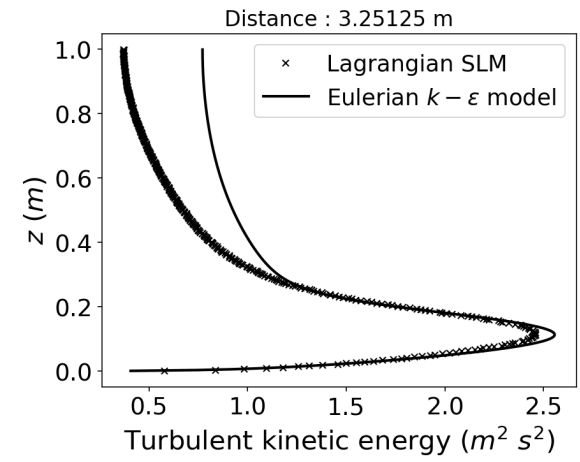

(f) $k-\epsilon$ model, downstream of the obstacle

Fig. 12 Reynolds stresses and turbulence kinetic energy vertical profiles at various distances from the inlet obtained with the hybrid Rotta model/SLM ((a), (c), (e)) and $k-\epsilon / \operatorname{SLM}$ ((b), (d), (f)) formulations, for the case of the flow around an obstacle within a boundary layer. The predictions correspond to the result of the Eulerian solver (continuous lines) and the Lagrangian one (crosses). With the Rotta model/SLM formulation, consistent duplicate predictions are obtained, whereas the $k-\epsilon /$ SLM formulation gives inconsistent predictions for the fluid kinetic energy 
First of all, it has been recalled that the respect of the well-mixed condition is ensured when the mean pressure-gradient term is correctly introduced as the mean drift term of Langevin models for the evolution of instantaneous fluid particle velocities. This has been demonstrated by numerical results obtained in various configurations that cover a range of challenging situations involving non-homogeneous, recirculating, and wall-bounded turbulent flows. These examples also confirm that serious discrepancies result when this term is absent (with zones of particle depletion and/or accumulation).

In particle-based models, the incompressibility constraint is translated into a double one: that the mean velocity derived from particle velocities be of zero divergence, and that the mean concentration derived from particle positions remain constant. In practical numerical implementations, this is reflected by a double correction, first with the introduction of the mean pressure-gradient term calculated from the zero-divergence condition of the mean flow and, second, by a correction step on particle positions. This formulation is valid for both stand-alone and hybrid Eulerian/Lagrangian numerical codes.

In practice, most applications for atmospheric dispersion studies are carried out with hybrid Eulerian/Lagrangian simulations. This framework has proven helpful for providing free-of-statistical-noise mean fluid properties (such as the mean velocity field) from the Eulerian solver to the Lagrangian one. However, hybrid formulations raise consistency issues for mean fluid properties predicted both from the Eulerian and Lagrangian models. It is therefore of paramount importance to realize that the development of improved Eulerian solvers, through advanced turbulence models, does not necessarily entail that Lagrangian predictions, based on a given Langevin model, will be improved. What is central is that both Eulerian and Lagrangian turbulence models be consistent in such hybrid formulations. In that sense, a second interest of the present work is to bring out this issue for atmospheric studies and to reveal, through numerical simulations in a boundary layer, the potentially-damaging errors induced when inconsistent turbulence models are used. The present model (coupled with a Rotta model for the Eulerian solver) thus serves as an illustration of how consistent hybrid formulations can be developed. Future works are needed to come up with Lagrangian models that are consitent with LES or Reynolds stress equation models.

It is hoped that the present results will be helpful to atmospheric models by allowing future theoretical and numerical efforts to be safely channeled towards improved predictions while ensuring that basic conditions and constraints are satisfied. Indeed, Lagrangian stochastic models have great interest for passive (as well as reactive) scalar modeling. For example, Bahlali et al. (2018) have considered point source dispersion and correctly captured the near- and far-field dispersive behaviors even when using a simple Langevin model. For future investigations, it will therefore be interesting to extend present consistent hybrid Eulerian/Lagrangian approaches to include scalar modeling based on more-advanced generalized Langevin model. 
Acknowledgements The authors would like to express special thanks to Jean-Pierre Minier for insightful advice and fruitful discussions.

The work of M. L. Bahlali was supported by CEREA, a member of the Pierre-Simon Laplace Institute (IPSL). The work of C. Henry was supported by the PRESTIGE Program (grant PRESTIGE-2017-1-0025) coordinated by Campus France. Through this PRESTIGE program, this research has received funding from the People Program (Marie Curie Actions) of the European Unions Seventh Framework Program (FP7/2007-2013) under REA grant agreement n. PCOFUND-GA-2013-609102.

\section{References}

Alessandrini S, Ferrero E (2009) A hybrid Lagrangian-Eulerian particle model for reacting pollutant dispersion in non-homogeneous non-isotropic turbulence. Physica A Stat Mech Appl 388(8):1375-1387

Archambeau F, Méchitoua N, Sakiz M (2004) Code Saturne: A Finite Volume Code for the computation of turbulent incompressible flows - Industrial applications. Int J Finite Volumes 1(1)

Bahlali ML, Dupont E, Carissimo B (2018) A hybrid CFD RANS/Lagrangian approach to model atmospheric dispersion of pollutants in complex urban geometries. Int J Environ and Pollut 64(1-3):74-89

Bernardin F, Bossy M, Chauvin C, Drobinski P, Rousseau A, Salameh T (2009) Stochastic downscaling method: application to wind refinement. Stoc Env Res Risk A 23(6):851-859

Borgas MS, Flesch TK, Sawford BL (1997) Turbulent dispersion with broken reflectional symmetry. J Fluid Mech 332:141-156

Cassiani M, Franzese P, Giostra U (2005a) A PDF micromixing model of dispersion for atmospheric flow. Part I: development of the model, application to homogeneous turbulence and to neutral boundary layer. Atmos Environ 39(8):1457-1469

Cassiani M, Franzese P, Giostra U (2005b) A PDF micromixing model of dispersion for atmospheric flow. Part II: application to convective boundary layer. Atmos Environ 39(8):1471-1479

Cassiani M, Radicchi A, Albertson JD (2007) Modelling of concentration fluctuations in canopy turbulence. Boundary-Layer Meteorol 122(3):655-681

Champagne FH, Pao YH, Wygnanski IJ (1976) On the two-dimensional mixing region. J Fluid Mech 74(2):209-250

Chauvin C, Bernardin F, Bossy M, Rousseau A (2010) Wind simulation refinement: some new challenges for particle methods. In: Progress in Industrial Mathematics at ECMI 2008, Springer, pp 765-770

Chibbaro S, Minier JP (2011) A note on the consistency of hybrid Eulerian/Lagrangian approach to multiphase flows. Int J Multiphas Flow $37(3): 293-297$

De Baas AF, Van Dop H, Nieuwstadt F (1986) An application of the Langevin equation for inhomogeneous conditions to dispersion in a convective boundary layer. Q J Roy Meteor Soc 112(471):165-180

Dreeben TD, Pope SB (1997) Probability density function and Reynolds-stress modeling of near-wall turbulent flows. Phys Fluids 9(1):154-163 
Franzese P (2003) Lagrangian stochastic modeling of a fluctuating plume in the convective boundary layer. Atmos Environ 37(12):1691-1701

Lin JC, Gerbig C (2013) How can we satisfy the well-mixed criterion in highly inhomogeneous flows? A practical approach. Lagrangian Modeling of the Atmosphere pp 59-70

Minier JP (2015) On Lagrangian stochastic methods for turbulent polydisperse two-phase reactive flows. Prog Energ Combust 50:1-62

Minier JP (2016) Statistical descriptions of polydisperse turbulent two-phase flows. Phys Rep 665:1-122

Minier JP, Peirano E (2001) The PDF approach to turbulent polydispersed two-phase flows. Phys Rep 352(1):1-214

Minier JP, Pozorski J (1995) Analysis of a PDF model in a mixing layer case. In: Proc. Tenth Symp. Turbulent Shear Flow, Pennsylvania State University, pp 26.25-26.30

Minier JP, Pozorski J (1999) Wall-boundary conditions in probability density function methods and application to a turbulent channel flow. Phys Fluids 11(9):2632-2644

Minier JP, Chibbaro S, Pope SB (2014) Guidelines for the formulation of Lagrangian stochastic models for particle simulations of single-phase and dispersed two-phase turbulent flows. Phys Fluids 26(11):113,303

Monin AS, Yaglom AM (1971) Statistical fluid dynamics. MIT Press, Cambridge

Peirano E, Chibbaro S, Pozorski J, Minier JP (2006) Mean-field/PDF numerical approach for polydispersed turbulent two-phase flows. Prog Energ Combust 32(3):315-371

Pope SB (1985) PDF methods for turbulent reactive flows. Prog Energ Combust 11(2):119-192

Pope SB (1987) Consistency conditions for random-walk models of turbulent dispersion. Phys Fluids 30(8):2374-2379

Pope SB (1991) Computations of turbulent combustion: progress and challenges. In: Symposium (International) on Combustion, Elsevier, vol 23, pp 591-612

Pope SB (1994a) Lagrangian PDF methods for turbulent flows. Annu Rev Fluid Mech 26(1):23-63

Pope SB (1994b) On the relationship between stochastic Lagrangian models of turbulence and second-moment closures. Phys Fluids 6(2):973-985

Pope SB (2000) Turbulent flows. Cambridge Univ. Press

Sawford B (1986) Generalized random forcing in random-walk turbulent dispersion models. Phys Fluids 29(11):3582-3585

Sawford BL (1985) Lagrangian statistical simulation of concentration mean and fluctuation fields. J Clim Appl Meteorol 24(11):1152-1166

Sawford BL, Guest FM (1988) Uniqueness and universality of Lagrangian stochastic models of turbulent dispersion. In: 8th Symposium on Turbulence and Diffusion, vol 1, pp 96-99

Stohl A, Forster C, Frank A, Seibert P, Wotawa G (2005) The Lagrangian particle dispersion model FLEXPART version 6.2. Atmos Chem Phys 
$5(9): 2461-2474$

Szewc K, Pozorski J, Minier JP (2012) Analysis of the incompressibility constraint in the smoothed particle hydrodynamics method. Int J Numer Meth Eng 92(4):343-369

Thomson DJ (1987) Criteria for the selection of stochastic models of particle trajectories in turbulent flows. J Fluid Mech 180:529-556

Tinarelli G, Mortarini L, Castelli ST, Carlino G, Moussafir J, Olry C, Armand P, Anfossi D (2013) Review and validation of MicroSpray, a Lagrangian particle model of turbulent dispersion. Lagrangian Modeling of the Atmosphere pp 311-328

Van Dop H, Nieuwstadt FTM, Hunt JCR (1985) Random walk models for particle displacements in inhomogeneous unsteady turbulent flows. Phys Fluids 28(6):1639-1653

Wacławczyk M, Pozorski J, Minier JP (2004) Probability density function computation of turbulent flows with a new near-wall model. Phys Fluids 16(5):1410-1422

Wilson JD, Sawford BL (1996) Review of Lagrangian stochastic models for trajectories in the turbulent atmosphere. Springer

Wilson JD, Thurtell GW, Kidd GE (1981) Numerical simulation of particle trajectories in inhomogeneous turbulence, III: Comparison of predictions with experimental data for the atmospheric surface layer. Boundary-Layer Meteorol 21(4):443-463 